\title{
Whole Slide Imaging (WSI) in Pathology: Current Perspectives and Future Directions
}

\author{
Neeta Kumar ${ }^{1} \cdot$ Ruchika Gupta ${ }^{2}$ (D) - Sanjay Gupta ${ }^{2}$ \\ Published online: 28 May 2020 \\ (C) Society for Imaging Informatics in Medicine 2020
}

\begin{abstract}
Whole slide imaging (WSI), ever since its first introduction about two decades ago, has been validated for a number of applications in the field of pathology. The recent approval of US FDA to a WSI system for use in primary surgical pathology diagnosis has opened avenues for wider acceptance and application of this technology in routine practice. The ongoing technological advances in digital scanners, image visualization methods, and the integration of artificial intelligence-derived algorithms with these systems provide opportunities of its newer applications. Its benefits are innumerable such as ease of access through internet, avoidance of physical storage space, and no risk of deterioration of staining quality or breakage of slides to name a few. Various barriers such as the high cost, technical glitches, and professional hesitation to adopt a new technology have hindered its use in pathology. This review article summarizes the technical aspects of WSI, its applications in diagnostic pathology, training, and research along with future perspectives. It highlights the benefits, limitations, and challenges delaying the use of this technology in routine practice. The review is targeted at students, residents, and budding pathologists to better acquaint them with the key aspects of state-of-the-art technology and enable them to implement WSI judiciously.
\end{abstract}

Keywords Whole slide imaging · Cytopathology $\cdot$ Telepathology $\cdot$ Diagnosis $\cdot$ Education $\cdot$ Automated image analysis · Validation $\cdot$ Regulation

\section{Introduction}

Digital imaging is widely used by pathologists for creation of static images using microscope-dedicated optical cameras and, more recently, using smartphones [1]. The introduction of whole slide imaging (WSI) in 1999 provided the opportunity of digitally converting the entire tissue on glass slide into a high-resolution virtual slide (VS). In the last two decades, we have witnessed an exponential growth in technology of acquiring virtual slide as well as its applications in various subspecialties of pathology [1].

Neeta Kumar and Ruchika Gupta contributed equally to this work.

Sanjay Gupta

sanjaydr17@gmail.com

1 Department of Pathology, Faculty of Dentistry, Jamia Millia Islamia (Central University), New Delhi 110025, India

2 Division of Cytopathology, ICMR-National Institute of Cancer Prevention and Research, I-7, Sector-39, Noida 201301, India
Development and refinement of artificial intelligence (AI) and machine learning algorithms have been an area of intense research in both radiology and pathology for automated or computer-aided diagnosis. WSI is a promising tool to develop and utilize such algorithms to be used in diagnostic pathology. There has been an ever-increasing demand for telemedicine which surged remarkably in times of lockdowns of the current corona pandemic throughout the globe. As the country prepares itself for telemedicine under the guidance of Medical Council of India [2], the authors have reviewed the existing literature as a part of preparedness for adopting the new technology in routine pathology, with special reference to cytopathology.

Since majority of the available studies pertain to application of WSI in surgical pathology, its utility in cytopathology has not been established well [3]. The authors of this review have been practicing cytopathology for last 3-4 decades and their personal experience has shown that conventional cytology slides pose difficulty in conversion to a good-quality WSI due to the presence of single cells and cell groups in different planes which necessitates repeated focusing. Hence, this review paper, besides encompassing the various aspects of WSI 
in diagnostic pathology in general, lays a special focus on the available literature on its applications and limitations in cytopathology subspecialty.

This review summarizes the technical aspects of WSI, status of current applications, and potential future applications in pathology. The benefits, limitations, and various challenges related to adoption of this technology in routine practice are discussed based on both review of the literature and personal experience of the authors [4]. This review is targeted at medical students, residents, and budding pathologists to apprise them of the basics of WSI technology and accompanying informatics. This is likely to help them make an informed decision related to WSI implementation.

\section{Technical Requirements for WSI}

WSI includes four sequential processes: image acquisition, storage, processing, and visualization. The hardware components of the device required for image acquisition comprise of two systems: image capture and image display. Image capture is performed by a digital scanner, which is basically a trinocular microscope with robotic control of illumination intensity, mechanical stage, objectives, and coarse and fine focusing facilities and is equipped with a high-resolution camera [5]. Unlike the still microscopic images, WSI scanners capture sequential images either in a tiled or line-scanning manner which are subsequently assembled or stitched into a VS, an exact replica of the glass slide [6]. For surgical pathology, the section thickness, placement of section in the center of slide, away from the coverslip edges, avoidance of artifacts of microtomy or mounting have to be optimized [1]. Thick slides or broken slides may not be scanned automatically, since most of the scanners accept only one slide-thick stacks. In this regard, use of liquid-based cytology (LBC) smear or cell blocks offer an advantage due to the standardized approach in preparation and staining.

Apart from the $x$ - and $y$-axes, digital scanners are required to utilize the $z$-axis of the slide in order to provide an image that appears similar to what we view through the microscope directly. The commercially available scanners differ in their methodology of z-focusing-from every tile to selected tiles or focus points [6]. The highest quality results in WSI are achieved by focusing on every tile during image acquisition, which is a time-consuming strategy leading to compromise in the throughput of the scanner.

The resolution of VS depends on the magnification used during scanning. Scanning at $\times 20$ magnification is considered suitable for routine surgical pathology and immunohistochemistry slides [7]. However, the same may not hold true for cytology slides. A study evaluating WSI in cervicovaginal cytology reported higher diagnostic accuracy with $\times$ 40 or $\times 40$ z-stack scanning [1]. For cytological samples, advanced cell layer topographical analysis can allow the samples to be selected for scanning based on the cell distribution on the smear. Such a triage helps both in speeding up the workflow and feedback to the laboratory for modification in collection of certain samples [8]. Continuous technological advances have made available high-throughput scanners (handling up to 400 slides), reduced scanning times (30 s to several minutes), and specialization for digitizing whole tissue mounts, larger glass slides, fluorescent-labeled sections, or smears [9].

The viewing and managing of VS is dictated by the intended use of the WSI system. Different vendors offer differing options such as image viewing software installed locally or as a part of software suite installed on network servers. These image viewers often provide the ability to annotate the image and export to other file formats. A few advanced image viewers such as Surface slide, Aperio ImageScope, and PathXL offer the ability of finger touch annotation using an onscreen virtual keyboard [10]. Use of devices such as smartphones, XDesk, or Microsoft Surfaces provides a large touch screen for interactive discussion of VS images in large meetings and conferences.

\section{Applications of WSI in Pathology}

Health care facilities are witnessing tremendous digitization with inclusion of digital imaging in medical specialities such as radiology connected to hospital information systems (HIS), laboratory information systems (LIS), picture archiving, and communication systems. Pathology laboratories equipped with WSI facility would gel well in such a setting with varied applications in diagnosis, education, and research.

\section{Digital Diagnosis, Teleconsultation, Ancillary Studies, Quality Assurance Programs, and Archiving Services}

Telepathology has been one of the first uses of WSI allowing a pathologist in a centralized laboratory to support peripheral centers in specialized sign-outs, and offering the sender pathologist an opportunity to seek experts' opinion on a case without incurring the expense or delay in international shipping $[4,11]$. Telepathology has been validated for second opinion in challenging cases of surgical pathology, cytopathology, and immunohistochemistry with the American Telemedicine Association issuing guidelines for the adoption of telepathology in patient care [12-15]. As a step forward, the United States' Food and Drug Administration (FDA) granted its approval, in 2017 to the first WSI (Philips IntelliSite Pathology Solution ${ }^{\mathrm{TM}}$ ) for primary diagnosis in surgical pathology on the basis of non-inferiority of WSI vis-à-vis glass slide with respect to diagnostic concordance and the reproducibility of repeated scanning [16, 17]. However, a review from the Digital Pathology Committee of the College of American Pathologists highlighted some key issues like the need for laboratories to internally validate WSI in their clinical practice 
and the opportunity for the pathologists to render diagnosis from locations other than their offices [18]. These queries need to be addressed by the regulatory authorities to ensure a responsible use of WSI with a view to strengthen the place of pathology in the modern era of precision medicine.

The other diagnostic applications of WSI in pathology have included intra-operative frozen section diagnosis through remote telepathology consultation with experts. A high concordance rate between WSI-based frozen section and permanent section diagnosis or on-site interpretation has been demonstrated in a number of studies [19, 20]. However, further studies on a range of pathologies from various organ systems are required to validate the utility and limitations of WSI in this field.

WSI offers added advantages in enhancing objectivity in the interpretation of immunohistochemistry and electron microscopy used in tumor diagnosis, prognosis, and evaluation of biomarkers for targeted therapy. A concordance of $90 \%$ between WSI and glass slides of Her2/neu expression in breast cancer was reported in a recent study [21]. Application of automated image analysis with algorithm-based scoring for the prognostic markers can assist in improving the scoring protocols and thereby enhance the efficacy of targeted therapies [20]. In electron microscopy, virtual ultrathin slide allows the pathologists to navigate the slide in their office while noting the exact location of the specific features. Apart from this, WSI technology can be valuable for obtaining consultation on ultrathin sections from experts located in higher centers [22].

WSI can also facilitate preparation and conduct of tumor boards through obviating the need of a mutiheaded microscope or microscope with projection attachment or acquisition of multiple static images of a case [23]. The use of this technology in quality assurance (QA) programs in surgical pathology and cytopathology can help in cost cutting and overcoming transportation difficulties, as also minimizing the potential second-reviewer bias by hiding the initial diagnosis [24]. Studies have also demonstrated the ease of same-day QA reviews with $>90 \%$ diagnostic agreement $[24,25]$.

The constant and ever-increasing requirement of physical space for storage of glass slides can also be taken care of with WSI and creation of VS. The routine H\&E slides as well as special stains, immunochemistry, or fluorescent-labeled slides can be digitally archived while still fresh and free from artifacts. Digital images, if linked to the HIS, shall be readily available in the electronic health record of a patient [26]. Similarly, WSI provides a permanent digital record for cases sent physically for consultation or tissue sent elsewhere for molecular testing, as also for medico-legal and forensic purposes [27].

\section{Pathology Education, Training, and Competency Testing}

The traditional practice of pathology training and examination through physical microscope-based sessions is witnessing a sea change with recent focus on the ability to diligently identify and interpret rather than the skill of using the microscope [28]. Since glass slides are a scarce resource with the case range dependent on the laboratory or center, WSI provides an opportunity of standardization of pathology education across the country with the same slides being viewed simultaneously by all students. WSI also allows the experts to impart training in interpretation of immunohistochemistry or electron microscopy to pathology residents in remote areas having limited or no exposure to these specialized fields. The WSI images can also be annotated and linked with relevant clinical and/or radiological data so as to provide a holistic view of the diagnostic approach to the students. Use of WSI also obviates the need of having large classrooms with numerous microscopes or multi-headers for teaching the students [29].

WSI offers many benefits to learners as well such as flexibility to engage at their own pace, compare normal and abnormal even on the same screen and foster a team-based learning spirit. The WSI collections can also be employed for pathology examinations and proficiency testing. For instance, the American Board of Pathology utilized $25 \mathrm{VS}$ along with 120 static digital images during a computer-based anatomic pathology examination [30]. Online WSI resources such as CAP Virtual Slide Box, Digital Pathology Associationhosted repository, and the Cancer Digital Slide Archive offer virtual slide sets for training and learning purposes [31-33]. Virtual slides are also being used in pathology conferences and meetings to promote interactive learning and provide ease of visualization of multiple images of different stains in conjunction with relevant clinical material [34].

Electronic publication of text books and articles in scientific journals has also opened new vistas of scientific communication [35]. Utilization of WSI-generated VS has proven to be the single most upgrade for pathology journals, thus empowering the readers to be involved in a scientifically based diagnostic approach to the lesion described [36].

\section{Research}

WSI has attracted the attention of biotechnology and pharmaceutical companies because of the opportunity to understand spatial relationship of various biological phenotypes and assistance in development of IHC-based biomarkers that can be utilized further in translational research studies [7]. In conjunction with tissue microarrays, WSI with image analysis tools allows the researchers to assess and score the biomarkers across all specimens quickly and objectively. In instances of possible biomarker heterogeneity, fluorescent WSI or multispectral imaging facilitates multiplexed analysis and supports further biomarker or drug discovery [37]. This technology can also be employed in development of oncologic biomarker strategies with augmented throughput and quantitative 
accuracy, hence supporting drug discovery [38]. Since advanced WSI scanners can function with transmitted light as well as fluorescent modes, their range of applications in research is radical.

\section{WSI in Cytopathology Subspecialty}

Compared with surgical pathology, the application of WSI in cytopathology is inherently difficult due to the threedimensional nature of the cytology smears. Hence, it is imperative to integrate $z$-stacking or multiplane scanning into the systems intended for use in cytopathology [3]. The z-stacking can be circumvented by multiplane scanning and use of the best focused image at each tile into the final file. Another approach that has been recently attempted includes the conversion of $z$-stacks of images into video frames and storing the stack as a high-efficiency video coding file. Subsequent video compression was shown to exceed the JPEG compression with comparable image quality [39].

There have been a few studies on the use of WSI in cytopathology. A comparison of conventional glass slides and WSI in 10 cervical and 20 non-gynecologic cytology cases showed similar diagnostic concordance between the two modalities among reviewing cytopathologists [40]. Another recent study comparing WSI with glass slides of thin-layer cervical specimens demonstrated $95.3 \%$ concordance rates, paving the way for WSI use in routine cytologic diagnosis [41]. Wright et al., in their study, evaluated the efficiency of WSI in cervico-vaginal cytology and highlighted issues such as a lack of familiarity with the technology, difficulty for the WSI in detecting few abnormal cells in the smears, problems with hyperchromatic crowded groups, and enormous image file size leading to increased scanning times. Another issue that is probably unique and intrinsic to cytology is the inability of the whole slide scanners to scan the edges of the coverslip [1].

The quality of WSI images when applied to cytology smears is fraught with certain problems that are not encountered in tissue sections, such as (a) presence of dense tissue fragments making it difficult for scanners to focus on the cells, (b) red cell contamination of the smear leading the scanner to focus on red cells in the background rather than the cells of interest, (c) smears with scant cellularity making z-stacking difficult, and (d) need to remove the screening marks/dots before scanning (for which keeping a photographic record of the diagnostic screening marks is recommended) [8]. Papstained smears, due to their wet fixation, often have cells in multiple planes and thus require $\mathrm{z}$-scanning to obtain a crisp and good-quality WSI image. On the other hand, air-dried Romanowsky-stained smears can be scanned with only $x$ and $y$-axes, since air drying flattens the cells thus minimizing the requirement of $z$-stacking [8].

\section{Challenges and Limitations of WSI}

WSI, with all its aforementioned advantages, holds promise in various arenas of pathology. However, it is prudent to acknowledge that many unresolved issues, as outlined below, still need to be addressed before WSI finds its place in routine application across the wide specialty of pathology.

\section{Cost}

The cost of procurement, implementation, and operational costs of WSI may be prohibitive, especially for small pathology laboratories due to huge initial cost of the scanners (US $\$ 100,000$ to US $\$ 1,500,000$ per piece) and additional hidden costs of training of staff and pathologists, technical support, digital slide storage systems, and regulatory or licensing costs [42]. Technological support for telepathology further compounds these costs. A recently published cost-benefit analysis at a large-volume academic center with slides in excess of 1.5 million showed a projected $\$ 1.3$ million savings over a 5 -year period [43]. However, the same analysis needs to be undertaken for smaller laboratories and low-resource settings.

\section{Technological Glitches}

While considering the implementation of WSI, it must be kept in mind that the WSI images would be only as good as the original glass slide. Scanning the whole slide/smear is a tedious and time-consuming process at present. Scanning times can vary from 1 to $5 \mathrm{~min}$ for a small biopsy to 5-20 $\mathrm{min}$ for a surgical specimen and 3-5 min for a liquid-based cytology smear $[8,42]$. This time can further go up to hour(s) for multiplane or z-stacked scanning. Another limitation with currently available scanners is the requirement of massive data storage capacity. Scanning at $\times 40$ magnification of a $1-\mathrm{mm}^{2}$ area results in a file size of 48 megabytes. Hence, majority of the WSI systems incorporate image compression algorithms (JPEG, JPEG 2000, LZW) to reduce the file size. However, image compression introduces image artifacts [7]. Some scanners offer the ability of multi-resolution representation (pyramid representation) where the field of view on the screen is inversely proportional to the magnification being viewed [7]. Majority of the WSI systems utilize a content management system (CMS) with specific programming in order to display the virtual slides in a consistent and specific manner [44].

Currently, there are vendor-dependent limitations with WSI systems. Some vendors use proprietary modules with limited scope of cross-browser compatibility or seamless execution on multiple devices. However, there are some opensource WSI systems such as cuMicroscope, Digital Slide Archive, the Sedeen viewer, and QuPath that support invocation of image analysis software [45]. 
Storage and confidentiality issues of the VS, especially when linked to the patients' metadata and clinical information, also need to be given due consideration. If retention of WSI data is necessary, reliable and safe archival with backup strategy such as off-site storage, RAID (redundant array of independent disks) storage, optical storage, or some combinations should be available [7].

\section{Professional Barriers}

Unlike radiology where digital systems obviate the need of making films, WSI in pathology does not reduce the laboratory's workload since glass slides still need to be prepared to be scanned. However, WSI does allow for streamlined navigation of the slides at various magnifications without the fear of accidentally breaking a slide at the microscope. The current WSI systems allow for batch-wise scanning of slides, thus improving the efficiency of the laboratory [7].

Other commonly encountered issues include available bandwidth of the network at the pathologists' workplace, security issues related to information technology, and installation of compatible browsers. However, with progress in information technology, the systems shall continue to be upgraded for improved speed and compatibility with browsers.

The USFDA approval of WSI in primary surgical pathology diagnosis does open up the issue of legal implications for the reporting pathologists, as discussed earlier. The relevant regulatory agencies (such as CLIA) need to put forth their guidelines in light of the expected changes with adoption of WSI by pathologists.

\section{Regulatory Issues}

Though USFDA has accorded its approval for use of WSI in surgical pathology practice, the other subspecialties of pathology still have a long path to tread towards this goal. At the same time, validation of WSI for introduction into the surgical pathology practice is still merely a recommendation of College of American Pathologists [18]. Regulations also need to be put in place regarding the archiving, retrieval, and access rights of the virtual slide library so formed.

Hence, the choice of the WSI system for a laboratory should consider factors such as the desired application, pertinent resolution to work upon, the time-toview (time from placing the slide on the scanner to viewing of the VS) and throughput, image analysis options, and the possibility of multiplane scanning and/or integration with the HIS or patient's electronic health record.

\section{Discussion}

As outlined in the present review, WSI is an exciting and promising technology with various advantages and a few challenges to be considered by the interested stakeholders. To overcome the biggest limitation, i.e., the cost or investment factor, numerous attempts have been made to develop costeffective WSI systems. For instance, a low-cost and highthroughput WSI (OpenWSI) built with open-source hardware and using single-frame autofocusing has been developed [46]. A scalable WSI with smartphones mounted on optical microscopes was developed for android as well as iPhone smartphones [47]. However, before recommending for use, these low-cost systems need to be validated for their quality, scanning reproducibility, and diagnostic accuracy on WSI images. Another challenge posed by vendor-specific modules can potentially hinder optimum utilization of this technology in applications such as telepathology. This can potentially be circumvented by use of open-source software such as Google Earth that requires minimal technical skills or assistance. The future of WSI lies in having an ideal vendor-neutral archive wherein a single software-hardware solution allows single viewing, storage, and retrieval with no barriers of the data source.

Validation of the WSI systems in different subspecialties of pathology and conduct of research with adequate sample size inclusive of all organ systems is also the need of the hour. Studies conducted so far have taken into account either a specific organ system or a limited number of cases of more than one body site. For laboratories with intention of incorporating WSI in routine surgical pathology sign-outs, it would be a good practice to undertake a validation study with a mix of the range of body sites and cases (benign and malignant) received by the laboratory.

Computer algorithms utilizing image analysis to recognize tumor areas, quantifying tissue features, delineating the tissue architecture, and cellular relationships in WSI images need to be developed and refined to offer computer-assisted diagnosis (CAD). Though CAD has been in vogue for cervical cytology since the late nineties with a number of commercially available systems offering screening and flagging of smears for pathologists' review [48], recent international initiatives utilized WSI with CADS in clinical diagnosis, such as Camelyon 17 challenge for a fully automated method to detect nodal metastasis of breast cancer in WSI images or the NIH-led Kidney Precision Medicine Project [49, 50].

\section{WSI in Pathology: Where Does Future Lie?}

1) Availability of high-resolution 3-dimensional imaging, especially for tumors, would improve the use of this technology with correlation between radiologic imaging and WSI. 
2) Multispectral imaging, when applied to WSI, would offer the ability to characterize chromatic properties and support color-based classification and multi-labeling studies.

3) Adoption of DICOM (Digital Imaging and Communications in Medicine) standards by the WSI vendors would allow vendor-neutral interoperability.

4) Refinement of artificial intelligence and machine learning algorithms would allow the pathologists contribute in a larger role in improving patient management and outcomes.

\section{Summary}

Virtual microscopy using whole slide scanning is an area of profound and rapid technologic development with far reaching and overarching applications in the field of pathology. Despite the advantages and claims of its non-inferiority compared with conventional microscopy, the adoption of this technique has been rather slow even in the developed nations. The barriers referred to in this paper currently preclude the wide application of whole slide scanning in the resource constrained medical institutions of the developing world. Some of these impediments may be overcome by collaborations between a reference laboratory equipped with a WSI system and smaller laboratories, through a hub-and-spoke model. Apart from the technical and cost-related issues, regulatory and validation requirements also need to be adequately addressed, especially for the developing nations. Nevertheless, virtual microscopy does provide a golden opportunity for pathologists to guide its evolution, standardization, and implementation by playing a key role in defining/ refining guidelines, designing the resource specific digital pathology laboratories, and propagating standardized educational modules to train the next generation of virtual pathologists.

\section{Compliance with Ethical Standards}

Conflict of Interest The authors declare that they have no conflict of interest.

Research Involving Human Participants and/or Animals Not applicable

Informed Consent Not applicable

\section{References}

1. Wright AM, Smith D, Dhurandhar B, Fairley T, Scheiber-Pacht M, Chakraborty S, Gorman BK, Mody D, Coffey DM: Digital slide imaging in cervicovaginal cytology: a pilot study. Arch Pathol Lab Med 137:618-624, 2013.
2. BOARD OF GOVERNORS (In supersession of the Medical Council of India). Telemedicine Practice Guidelines: Enabling Registered Medical Practitioners to Provide Healthcare Using Telemedicine. Available at https://www.mohfw.gov.in/pdf/ Telemedicine.pdf. Accessed 01 April 2020.

3. Wilbur DC: Digital cytology: current state of the art and prospects for the future. Acta Cytol 55:227-238, 2011.

4. Kumar N, Busarla SV, Sayed S, Kirimi JM, Okiro P, Gakinya SM, Moloo Z, Sohani AR: Telecytology in East Africa: a feasibility study of forty cases using a static imaging system. J Telemed Telecare 18:7-12, 2012.

5. Bueno G, Déniz O, Fernández-Carrobles Mdel M, Vállez N, Salido $\mathrm{J}$ : An automated system for whole microscopic image acquisition and analysis. Microsc Res Tech 77:697-713, 2014.

6. Indu M, Rathy R, Binu MP: "Slide less pathology": Fairy tale or reality? J Oral Maxillofac Pathol 20:284-288, 2016.

7. Zarella MD, Bowman D, Aeffner F, Farahani N, Xthona A, Absar SF, Parwani A, Bui M, Hartman DJ: A Practical Guide to Whole Slide Imaging: A White Paper From the Digital Pathology Association. Arch Pathol Lab Med 143:222-234, 2019.

8. Van Es SL, Greaves J, Gay S, Ross J, Holzhauser D, Badrick T: Constant Quest for Quality: Digital Cytopathology. J Pathol Inform 9:13, 2018.

9. Farahani N, Parwani A, Pantanowitz L: Whole slide imaging in pathology: advantages, limitations, and emerging perspectives. Pathol Lab Med Int 7:23-33, 2015.

10. Wang Y, Williamson KE, Kelly PJ, James JA, Hamilton PW: SurfaceSlide: a multitouch digital pathology platform. PLoS One.7:e30783, 2012.

11. Ghosh A, Brown GT, Fontelo P: Telepathology at the Armed Forces Institute of Pathology: A Retrospective Review of Consultations From 1996 to 1997. Arch Pathol Lab Med 142: 248-252, 2018.

12. Sawai T, Uzuki M, Kamataki A, Tofukuji I: The state of telepathology in Japan. J Pathol Inform. 1. 13, 2010.

13. Rodriguez-Urrego PA, Cronin AM, Al-Ahmadie HA, Gopalan A, Tickoo SK, Reuter VE, Fine SW: Interobserver and intraobserver reproducibility in digital and routine microscopic assessment of prostate needle biopsies. Hum Pathol 42:68-74, 2011.

14. Wilbur DC, Madi K, Colvin RB, Duncan LM, Faquin WC, Ferry JA, Frosch MP, Houser SL, Kradin RL, Lauwers GY, Louis DN, Mark EJ, Mino-Kenudson M, Misdraji J, Nielsen GP, Pitman MB, Rosenberg AE, Smith RN, Sohani AR, Stone JR, Tambouret RH, Wu CL, Young RH, Zembowicz A, Klietmann W: Whole-slide imaging digital pathology as a platform for teleconsultation: a pilot study using paired subspecialist correlations. Arch Pathol Lab Med. 133:1949-1953, 2009.

15. Evans AJ, Krupinski EA, Weinstein RS, Pantanowitz L: 2014 American Telemedicine Association clinical guidelines for telepathology: Another important step in support of increased adoption of telepathology for patient care. J Pathol Inform 6:13, 2015.

16. US Food \& Drug Administration. FDA news release: FDA allows marketing of first whole slide imaging system for digital pathology. https://www.fda.gov/newsevents/newsroom/pressannouncements/ ucm552742.htm. Accessed 01 April 2020.

17. Mukhopadhyay S, Feldman MD, Abels E, Ashfaq R, Beltaifa S, Cacciabeve NG, Cathro HP, Cheng L, Cooper K, Dickey GE, Gill RM, Heaton RP Jr, Kerstens R, Lindberg GM, Malhotra RK, Mandell JW, Manlucu ED, Mills AM, Mills SE, Moskaluk CA, Nelis M, Patil DT, Przybycin CG, Reynolds JP, Rubin BP, Saboorian MH, Salicru M, Samols MA, Sturgis CD, Turner KO, Wick MR, Yoon JY, Zhao P, Taylor CR: Whole Slide Imaging Versus Microscopy for Primary Diagnosis in Surgical Pathology: A Multicenter Blinded Randomized Noninferiority Study of 1992 Cases (Pivotal Study). Am J Surg Pathol 42:39-52, 2018. 
18. Evans AJ, Bauer TW, Bui MM, Cornish TC, Duncan H, Glassy EF, Hipp J, McGee RS, Murphy D, Myers C, O’Neill DG, Parwani AV, Rampy BA, Salama ME, Pantanowitz L: US Food and Drug Administration Approval of Whole Slide Imaging for Primary Diagnosis: A Key Milestone Is Reached and New Questions Are Raised. Arch Pathol Lab Med 142:1383-1387, 2018.

19. Huang Y, Lei Y, Wang Q, Li D, Ma L, Guo L, Tang M, Liu G, Yan Q, Shen L, Tong G, Jing Z, Zhang Y, Deng Y: Telepathology consultation for frozen section diagnosis in China. DiagnPathol. 13:29, 2018

20. Fallon MA, Wilbur DC, Prasad M: Ovarian frozen section diagnosis: use of whole-slide imaging shows excellent correlation between virtual slide and original interpretations in a large series of cases. Arch Pathol Lab Med 134:1020-1023, 2010.

21. Boroujeni AM, Dehghani A, Yousefi E, Gupta R: Whole Slide Imaging for HER2/neu Immunohistochemistry: Reproducibility Study for Digital and Paired Glass Slide Interpretation. Am J Clin Pathol 146(suppl 1):36, 2016.

22. Lee KC, Mak LS. Virtual electron microscopy: a simple implementation creating a new paradigm in ultrastructural examination. Int $\mathrm{J}$ Surg Pathol 19:570-575, 2011.

23. Chen ZW, Kohan J, Perkins SL, Hussong JW, Salama ME: Webbased oil immersion whole slide imaging increases efficiency and clinical team satisfaction in hematopathology tumor board. J Pathol Inform 5:41, 2014.

24. Graham AR, Bhattacharyya AK, Scott KM, Lian F, Grasso LL, Richter LC, Carpenter JB, Chiang S, Henderson JT, Lopez AM, Barker GP, Weinstein RS: Virtual slide telepathology for an academic teaching hospital surgical pathology quality assurance program. Hum Pathol 40:1129-1136, 2009.

25. Ho J, Parwani AV, Jukic DM, Yagi Y, Anthony L, Gilbertson JR: Use of whole slide imaging in surgical pathology quality assurance: design and pilot validation studies. Hum Pathol 37:322-331, 2006.

26. Huisman A, Looijen A, van den Brink SM, van Diest PJ: Creation of a fully digital pathology slide archive by high-volume tissue slide scanning. Hum Pathol 41:751-757, 2010.

27. Pantanowitz L, Valenstein PN, Evans AJ, Kaplan KJ, Pfeifer JD, Wilbur DC, Collins LC, Colgan TJ: Review of the current state of whole slide imaging in pathology. J Pathol Inform.2:36, 2011.

28. Pratt RL: Are we throwing histology out with the microscope? A look at histology from the physician's perspective. Anat Sci Educ. 2:205-209, 2009.

29. Dee FR: Virtual microscopy in pathology education. Hum Pathol 40:1112-1121, 2009.

30. American Board of Pathology. http://abpath.org/index.php/takingan-examination/primary-certificate-requirements.Accessed 01 April 2020.

31. USCAP. http://syndrme.org/index.php/syndrme-resources/25pathology/124-uscap-virtual-slide-box. Accessed 01 April 2020.

32. Digital Pathology Association. https://digitalpathologyassociation. org/whole-slide-imaging-repository.Accessed 01 April 2020.

33. TCGA. http://cancer.digitalslidearchive.net. Accessed 01 April 2020.

34. Kumar N, Chandra M: Whole Slide Imaging in Medical and Dental Education. J Med Erudite 1:46-51, 2013.

35. Cagle PT, Glassy EF: Whole slide images add value to journal article figures. Arch Pathol Lab Med 138:592, 2014.

36. Glassy EF: Rebooting the Pathology Journal: Learning in the Age of Digital Pathology. Arch Pathol Lab Med 138:728-729, 2014.

37. Aeffner F, Zarella MD, Buchbinder N, Bui MM, Goodman MR, Hartman DJ, Lujan GM, Molani MA, Parwani AV, Lillard K, Turner OC, Vemuri VNP, Yuil-Valdes AG, Bowman D:
Introduction to Digital Image Analysis in Whole-slide Imaging: A White Paper from the Digital Pathology Association. J Pathol Inform 10:9, 2019.

38. Shinde V, Burke KE, Chakravarty A, Fleming M, McDonald AA, Berger A, Ecsedy J, Blakemore SJ, Tirrell SM, Bowman D: Applications of pathology-assisted image analysis of immunohistochemistry-based biomarkers in oncology. Vet Pathol 51:292-303, 2014.

39. Zarella MD, Jakubowski J: Video compression to support the expansion of whole-slide imaging into cytology. J Med Imaging (Bellingham). 6:047502, 2019.

40. Hanna MG, Monaco SE, Cuda J, Xing J, Ahmed I, Pantanowitz L: Comparison of glass slides and various digital-slide modalities for cytopathology screening and interpretation. Cancer Cytopathol 125:701-709, 2017.

41. Bongaerts O, Clevers C, Debets M, Paffen D, Senden L, Rijks K, Ruiten L, Sie-Go D, van Diest PJ, Nap M: Conventional Microscopical versus Digital Whole-Slide Imaging-Based Diagnosis of Thin-Layer Cervical Specimens: A Validation Study. J Pathol Inform 9:29, 2018.

42. Ghaznavi F, Evans A, Madabhushi A, Feldman M. Digital imaging in pathology: whole-slide imaging and beyond. Annu Rev Pathol 8: 331-359, 2013.

43. Hanna MG, Reuter VE, Samboy J, England C, Corsale L, Fine SW, Agaram NP, Stamelos E, Yagi Y, Hameed M, Klimstra DS, Sirintrapun SJ: Implementation of Digital Pathology Offers Clinical and Operational Increase in Efficiency and Cost Savings. Arch Pathol Lab Med 143:1545-1555, 2019.

44. Rocha R, Vassallo J, Soares F, Miller K, Gobbi H: Digital slides: present status of a tool for consultation, teaching, and quality control in pathology. Pathol Res Pract 205:735-741, 2009.

45. Pantanowitz L, Sharma A, Carter AB, Kurc T, Sussman A, Saltz J: Twenty Years of Digital Pathology: An Overview of the Road Travelled, What is on the Horizon, and the Emergence of VendorNeutral Archives. J Pathol Inform 9:40, 2018.

46. Guo C, Bian Z, Jiang S, Murphy M, Zhu J, Wang R, Song P, Shao $\mathrm{X}$, Zhang Y, Zheng G: OpenWSI: a low-cost, high throughput whole slide imaging system via single-frame autofocusing and open-source hardware. Opt Lett 45:260-263, 2020.

47. Yu H, Gao F, Jiang L, Ma S: Development of a Whole Slide Imaging System on Smartphones and Evaluation With Frozen Section Samples. JMIR Mhealth Uhealth 5:e132, 2017

48. Kumar N, Jain S: Quality control and automation in cervical cytology. J Indian Med Assoc. 102:372, 374, 376, 2004.

49. Bandi P, Geessink O, Manson Q, Van Dijk M, Balkenhol M, Hermsen M, EhteshamiBejnordi B, Lee B, Paeng K, Zhong A, Li Q, Zanjani FG, Zinger S, Fukuta K, Komura D, Ovtcharov V, Cheng S, Zeng S, Thagaard J, Dahl AB, Lin H, Chen H, Jacobsson L, Hedlund M, Cetin M, Halici E, Jackson H, Chen R, Both F, Franke J, Kusters-Vandevelde H, Vreuls W, Bult P, van Ginneken B, van der Laak J, Litjens G: From Detection of Individual Metastases to Classification of Lymph Node Status at the Patient Level: The CAMELYON17 Challenge. IEEE Trans Med Imaging. 38:550-560, 2019.

50. National Institutes of Health, UK. https://www.niddk.nih.gov/ research-funding/research-programs/kidney-precision-medicineproject-kpmp. Accessed 01 April 2020.

Publisher's Note Springer Nature remains neutral with regard to jurisdictional claims in published maps and institutional affiliations. 